\title{
Further experimental data supporting the safety of potassium polyaspartate used as a food additive in wine stabilization
}

\author{
F. Colombo ${ }^{1}$, C. Di Lorenzo ${ }^{1}$, C. Casalegno ${ }^{2}$, G. Triulzi ${ }^{3}$, S. Marroncelli ${ }^{2}$, S. Biella $^{1}$, F. Orgiu ${ }^{1}$, and P. Restani ${ }^{1}$ \\ ${ }^{1}$ Dipartimento di Scienze Farmacologiche e Biomolecolari, Università degli Studi di Milano, Milan, Italy \\ ${ }^{2}$ ChemService, Novate Milanese (MI), Italy \\ ${ }^{3}$ Esseco S.r.1., Trecate (NO), Italy
}

\begin{abstract}
Potassium polyaspartate (KPPA) is a food additive used for wine stabilization. KPAA was included in the list of additives allowed in EU, with the Commission Regulation (EU) 2017/1399, having received a positive opinion by EFSA (European Food Safety Authority) in 2016. KPAA is rich in negative charges, which are essential for its enological function consisting in binding positive ions in order to reduce wine instability. Concerns were raised on the fact that the same binding properties could be responsible for a reduction of microelement bioavailability in humans. For this reason and for the protection of consumers' health, the binding properties of potassium polyaspartate versus three minerals (calcium, iron, magnesium) was assayed using the Size-Exclusion Chromatography (SEC). Aliquots obtained by SEC were analysed for their content in polyaspartate (by microbiuret method) and bound minerals (by ICP-OES method). The results obtained by this research shows that, when potassium polyaspartate is added to wine, the negative charges of the additive are saturated, as expected by the specific role of KPAA in tartaric stabilization. In conclusions, the effect on mineral bioavailability must be considered negligible.
\end{abstract}

\section{Introduction}

Although it does not affect the quality of wine, the presence of potassium bitartrate (KHT) deposits can negatively influence its acceptability by consumers [1]. To avoid the phenomenon, most wines are treated in order to eliminate the excess of tartaric acid and/or potassium to prevent the precipitation of KHT. The most usual solutions are the subtractive techniques (such as cold stabilization) [2] or the use of additives. Different molecules are allowed as stabilizing additives (metatartaric acid, cellulose gums and mannoproteins), but all of them show limits in their performance. In this contest the European Community financed the Project "Use of biopolymers for sustainable stabilization of quality wines (STABIWINE)", whose objective was the identification of new additives and their practical application. During the EU project several molecules were considered and, among others, potassium polyaspartate showed stabilizing properties similar to those of metatartaric acid, with an improved stability over time [1]. Potassium polyaspartate is the potassium salt of polyaspartic acid; it is synthesized from L-aspartic acid and potassium hydroxide.

From the toxicological point of view, potassium polyaspartate (KPAA) must be considered safe, being negative in the bacterial reverse mutation assay (OECD $471,1997)$ and in the in vitro mammalian cell micronucleus test (OECD TG 487, 2008). Moreover, in a 90-day oral toxicity study performed in male and female Wistar rats (OECD TG 408), the no observed adverse effect level (NOAEL) was set at $1000 \mathrm{mg} / \mathrm{kg}$ bw per day, the highest dose tested [3].
Considering these data, the safety of potassium polyaspartate as a food additive was evaluated by EFSA (European Food Safety Authority); in its opinion dated March 9, 2016 [4] the Authority concluded that "there was no safety concern from the proposed use in wine at a maximum use level of $300 \mathrm{mg} / \mathrm{L}$ and typical levels in the range of $100-200 \mathrm{mg} / \mathrm{L}$ ". On the basis of this opinion, KPAA has been included in the list of additives allowed in EU, according to the Commission Regulation (EU) 2017/1399. During the application for approval in other world areas, further details were required about the possible interaction between polyaspartate consumed with wine and nutrients (minerals) from the diet. The reason of this request was the high number of free carboxylic groups present in KPAA, which could bind positively charged minerals and determine a reduction of their bioavailability.

To evaluate the interference of KPAA with metals bioavailability, the binding properties of KPAA was tested with three minerals: calcium, iron, magnesium. They were considered the most suitable nutrients to develop a good model of the phenomenon considering their nutritional characteristics (frequent deficiency and/or low bioavailability).

\section{Materials and methods}

In order to evaluate the interaction between potassium polyaspartate and minerals, a solution of KPAA was firstly incubated with a known amount of each mineral (calcium, iron and magnesium) and afterwards the solution was loaded onto a column for Size-Exclusion Chromatography (SEC). Aliquots of the eluted solution were then dosed 
to quantify the amount of polyaspartic acid (PAA) by microbiuret method and the amount of bound mineral by ICP-OES method.

\subsection{KPAA stock solutions}

Potassium polyaspartate used in this study had the chemical characteristics listed in Table 1. Two different stock solutions were prepared:

- KPAA stock solution $\mathrm{n}^{\circ} 1-10.5 \mathrm{mg} / \mathrm{mL}$ KPAA $(9.96 \mathrm{mg} / \mathrm{mL}$ as dry weight) in water;

- KPAA stock solution $\mathrm{n}^{\circ} 2-10 \mathrm{mg} / \mathrm{mL}$ KPAA $(9.46 \mathrm{mg} / \mathrm{mL}$ as dry weight) in Phosphate Buffered Saline solution (PBS) $\left(10 \mathrm{mM} \mathrm{Na} \mathrm{HPO}_{4}, 1.76 \mathrm{mM}\right.$ $\mathrm{NaH}_{2} \mathrm{PO}_{4}, 139.7 \mathrm{mM} \mathrm{NaCl}$ - $\mathrm{pH}$ 7.4).

\subsection{Size-exclusion chromatography}

Due to their difference in molecular weight, PAA, without or with bound metals) and free minerals were separated by Size-Exclusion Chromatography (SEC). The chromatographic column was prepared using Sephadex G-15 (Pharmacia, Sweden), which has a separation range between 500 and $1500 \mathrm{Da}$. KPAA has a MW close to $5 \mathrm{kDa}$, therefore it is totally excluded from the gel pores and eluted with the void volume (Vo).

On the contrary, having a MW lower than $500 \mathrm{Da}$, all free minerals are eluted with the total volume of the column (Vt).

Sephadex G-15 is supplied as a dry powder, and must be allowed to swell in PBS, before use. A quantity of $6.7 \mathrm{~g}$ of Sephadex G-15 was weighed and added to $20 \mathrm{~mL}$ of PBS for a swelling time of 3 hours. Then, the suspension was degassed under vacuum to eliminate gas bubbles and the column was packed by gravity. The column had a height of $10 \mathrm{~cm}$ and internal diameter of $1.5 \mathrm{~cm}$ $(\mathrm{Vt}=20 \mathrm{~mL} ; \mathrm{Vo}=$ approximately $7 \mathrm{~mL})$. Aliquots of $1 \mathrm{~mL}$ of samples were loaded onto the packed column. Volumes of $1 \mathrm{~mL}$ were collected during elution and monitored at $280 \mathrm{~nm}$ by a UV-visible spectrophotometer (Varian Cary 50 SCAN, Palo Alto, California, USA). The aliquots close to Vo were quantified for their PAA concentration by microbiuret method. In parallel, the concentration of the three metals considered was quantified by Inductively Coupled Plasma - Optical Emission Spectrometry (ICP-OES) method.

\subsection{Quantification of polyaspartate (PAA) by microbiuret method}

This assay quantifies proteins/polypeptides thanks to a specific chemical reaction between peptidic bonds in the polypeptidic sequence and an alkaline copper sulfate reagent [5]. The absorbance, measured at $310 \mathrm{~nm}$ (Varian Cary 50 SCAN, Palo Alto, California, USA), is directly proportional to the concentration of peptidic bonds. For its specific amino acidic composition, it was not possible to quantify polyaspartate using (as usual) bovine serum albumin (BSA) as a protein reference standard. PAA was quantified by using a linear regression obtained with increasing concentrations of KPAA stock solution n. $2(0.048-0.95 \mathrm{mg} / \mathrm{mL})$. Aliquots of $2 \mathrm{~mL}$ of samples (suitably diluted) or standards were added in parallel with: A) $\mathrm{mL}$ of microbiuret reagent $\mathrm{n} .1(0.2 \%$ copper
Table 1. Chemical characteristics of KPAA.

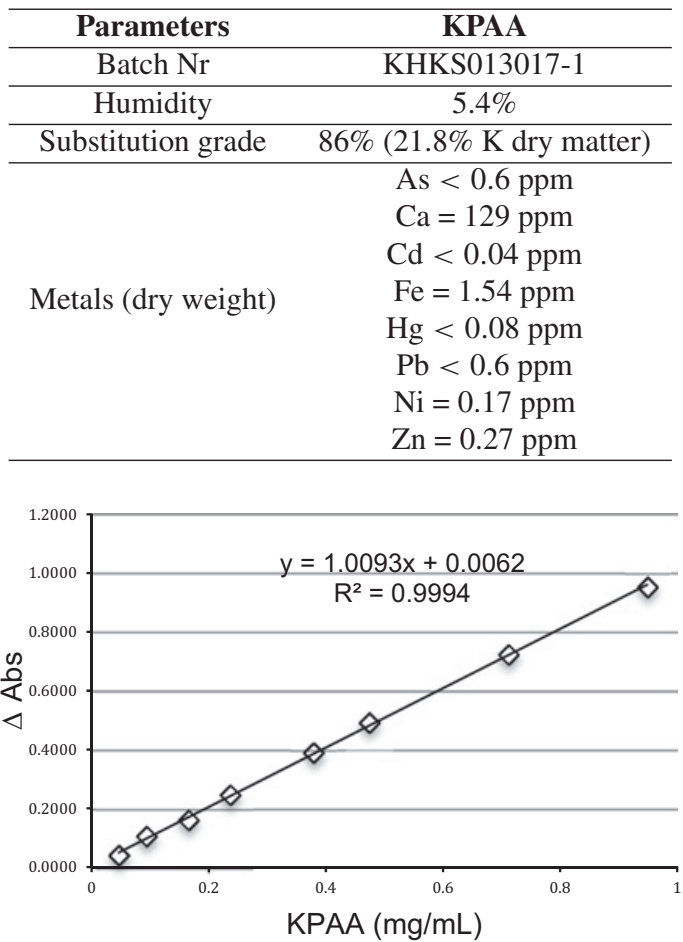

Figure 1. Linear regression used to quantify PAA in samples by microbiuret method.

sulfate (w/v) in $30 \% \mathrm{NaOH})$, or B) $1 \mathrm{~mL}$ of microbiuret reagent n. $2(30 \% \mathrm{NaOH})$. The absorbance at $310 \mathrm{~nm}$ of the samples treated with reagent $\mathrm{B}$ was subtracted by those obtained by addition of solution $\mathrm{A}$ to eliminate possible interferences (haze). A stock solution of KPAA in water (stock solution $\mathrm{n}^{\circ} 1-9.96 \mathrm{mg} / \mathrm{mL}$ ) was used as a blank.

\subsection{Quantification of minerals by ICP-OES}

The samples preparation and the quantification of minerals were performed using ICP-OES, accordind to the ESSECO internal procedures.

\subsubsection{Sample preparation}

Potassium (K), Sodium (Na), Magnesium (Mg), and Calcium (Ca)

In order to quantify $\mathrm{K}, \mathrm{Na}, \mathrm{Mg}$ and $\mathrm{Ca}$, the samples obtained from the SEC elution were diluted 1:200 (0.1 mL in $20 \mathrm{~mL}$ of elution solution) and added with the internal standard.

Since elution aliquots obtained after KPAA incubation with $\mathrm{Fe}$ showed a precipitate, a different sample preparation was applied:

- 4 grams of precipitate were weighted with an analytical balance $(1.0 \mathrm{mg}$ precision) in a calibrated porcelain crucible.

- Sample was maintained 6 hours in the oven at $105^{\circ} \mathrm{C}, 1$ hour at $300^{\circ} \mathrm{C}$ on an heated plate and finally in muffle furnace at $600^{\circ} \mathrm{C}$ for one night.

- The ashes were suspended with $20 \mathrm{~mL}$ of ultrapure water and $2 \mathrm{~mL}$ of ultrapure chloridric acid on a heated plate at $90^{\circ} \mathrm{C}$ for 2 minutes. 


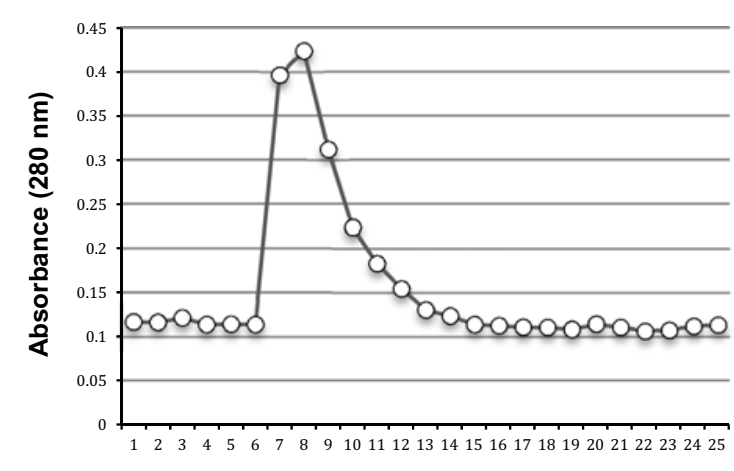

Aliquots

Figure 2. Elution profile of PAA after incubation with $\mathrm{CaCl}_{2}$.

- The sample was cooled and brought to volume into a $50 \mathrm{~mL}$ volumetric flask.

- The internal standard was added and the sample was analysed by ICP-OES.

\subsubsection{Instrumental analysis based on ICP-OES equipment}

The equipment and the operating conditions of ICP-OES analysis were:

- Radial Spectrometer ICP-OES. GENESIS model;

- Instrument operating conditions: Schott chamber, Cross-Flow standard nebulizer;

- Calibration (0-500 ppm) was set up for $\mathrm{K}^{+}, \mathrm{Na}^{+}$, $\mathrm{Mg}^{++}, \mathrm{Ca}^{++}$

- Calibration (0-5 ppm) was set up for $\mathrm{Fe}^{3+}$ with multi element standard;

- Analytical standards were solubilized in $4 \%$ nitric acid solution;

- Yttrium was used as an internal standard for check;

- The software calculated the final concentration taking into consideration the dilution and weight.

\section{Results and discussion}

\subsection{Linear regression for the quantification of PAA}

KPAA, having the chemical characteristics reported in Table 1, was used as a standard to prepare the linear regression and quantify samples for their content in PAA before and after the size-exclusion chromatography. The linearity obtained was highly satisfactory $\left(\mathrm{R}^{2}\right.$ close to 1.0$)$, as shown in Fig. 1.

\subsection{Binding properties of KPAA vs. Calcium}

\subsubsection{Incubation procedure}

A volume of $5 \mathrm{~mL}$ of KPAA stock solution n. 2 was incubated with $\mathrm{CaCl}_{2}$ (15.5 mg, containing $5.59 \mathrm{mg}$ of $\mathrm{Ca}^{++}$) for 4 hours under stirring and then loaded onto the column for Size-Exclusion Chromatography to separate PAA (with or without bound metals) from free minerals.

Elution was monitored spectrophotometry at $280 \mathrm{~nm}$, collecting eluate aliquots of $1 \mathrm{~mL}$ each: collection started at loading and continued till the 25th aliquot (Vt). Fig. 2 shows the absorbance profile of SEC elution.
Considering the elution profile, the aliquots 5-12 contained molecules absorbing at $280 \mathrm{~nm}$ (proteins and peptides), confirming that PAA was eluted at Vo (MW > 1500 Da), being excluded by gel pores. These aliquots were collected in a unique sample and further investigated for protein (PAA) concentration by microbiuret method and for metal concentration by ICP-OES. The fraction containing free minerals, corresponding to the $\mathrm{Vt}$, was eliminated and not analysed.

\subsubsection{PAA concentration}

Using the linear regression illustrated above (Fig. 1), the protein concentration in the sample 5-12 was $1.206 \pm 0.083 \mathrm{mg} / \mathrm{mL}$; considering the total volume of this sample $(8 \mathrm{~mL})$, the $\mathrm{mg}$ of PAA recovered were $9.65( \pm 0.66)$, representing $102 \%$ of the expected amount $(9.46 \mathrm{mg})$.

\subsubsection{Calcium exchange}

Table 2 lists the concentration of metals in sample 5-12.

\subsubsection{Discussion}

The presence of $\mathrm{Na}$ is associated with the buffer used for the incubation of KPAA with $\mathrm{CaCl}_{2}$, and for its elution by SEC; as a consequence, its value was easily calculated and was similar in all samples apart from the KPAA Stock solution n. 1 (prepared in water). Sodium concentrations were in agreement with the expected ones (in brackets), when the calculation was possible.

The sample containing aliquots 5-12 showed calcium concentration lower than those of the incubation solution and this is partially due to the dilution during the sizeexclusion chromatography (the dilution is close to $10 \mathrm{x}$ ). Moreover, sample containing aliquots 5-12 showed a percentage of bound calcium ( $\mathrm{Ca} / \mathrm{PAA})$ lower than the ratio calculated in the incubation solution (8.2 vs. 10.5\%). This means that the quantity of Ca present in the incubation solution exceeded the exchange possibility of PAA and the highest amount of calcium that can be exchanged with K, corresponds to $8.2 \%$ of PAA weight.

\subsection{Binding properties of KPAA vs. Magnesium}

\subsubsection{Incubation procedure}

A volume of $5 \mathrm{~mL}$ of KPAA stock solution $\mathrm{n} .2$ was incubated with $\mathrm{MgCl}_{2} \times 6 \mathrm{H}_{2} \mathrm{O}(28.4 \mathrm{mg}$, containing $3.39 \mathrm{mg}$ of $\mathrm{Mg}^{++}$) for 4 hours under stirring and then loaded onto the column for SEC to separate PAA (with or without bound metals) from free minerals.

Elution was monitored spectrophotometry at $280 \mathrm{~nm}$, collecting eluate aliquots of $1 \mathrm{~mL}$ each; collection started at loading and continued till the 25th aliquot (Vt).

Fig. 3 shows the elution profile of size-exclusion chromatography.

As in the previous incubation assay, the aliquots 5-12 contained molecules absorbing at $280 \mathrm{~nm}$ (proteins and peptides) confirming that PAA was eluted at Vo. These aliquots were collected in a unique sample and further investigated for protein (PAA) concentration by microbiuret method and for metal concentration by ICPOES. 
Table 2. Ca and Na quantification in the sample 5-12^.

\begin{tabular}{cccccc}
\hline Samples & PAA $(\mathbf{m g} / \mathbf{L})$ & $\mathbf{N a}(\mathbf{m g} / \mathbf{L})$ & $\mathbf{C a}(\mathbf{m g} / \mathbf{L})$ & $\mathbf{N a} / \mathbf{P A A} \%$ & $\mathbf{C a} / \mathbf{P A A} \%$ \\
\hline KPAA stock solution 1 & 9960 & 665 & 4.5 & 6.68 & $0.045(0.014)$ \\
\hline KPAA stock solution 2 & 9460 & 3911 & 15.5 & $41.3(39.2)$ & $0.16(0.014)$ \\
\hline KPAA stock solution 2 with added $\mathrm{CaCl}_{2}$ & 9460 & 4022 & 996 & $42.5(39.2)$ & $10.5(11.8)$ \\
\hline Aliquots 5-12 & 1206 & 4011 & 98.7 & $332.6(308)$ & 8.2 \\
\hline
\end{tabular}

${ }^{\wedge}$ In brackets the expected values

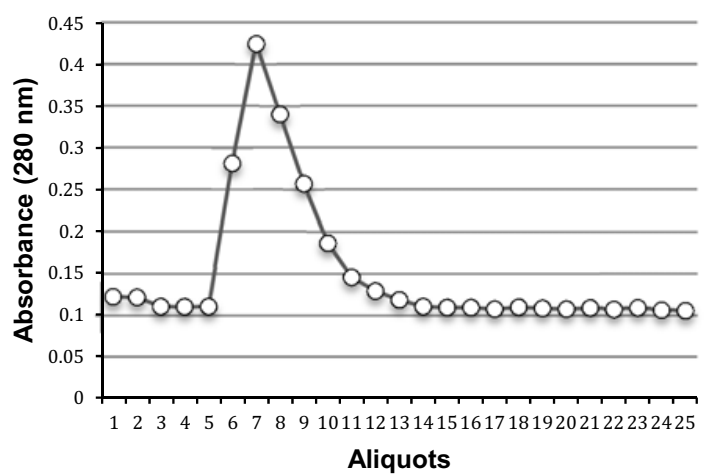

Figure 3. Elution profile of PAA after incubation with $\mathrm{MgCl}_{2}$.

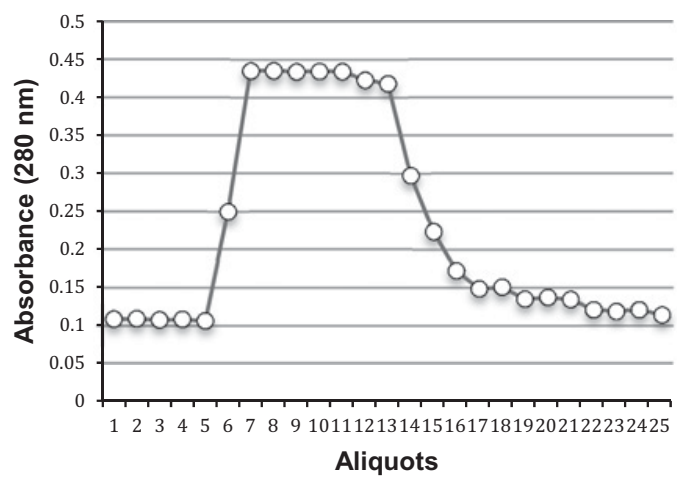

Figure 4. Elution profile of PAA after incubation with $\mathrm{FeSO}_{4}$

\subsubsection{PAA concentration}

The concentration of PAA in samples 5-12 was $1.063 \pm$ $0.108 \mathrm{mg} / \mathrm{mL}$; considering the total volume $(8 \mathrm{~mL})$, the $\mathrm{mg}$ of PAA recovered were $8.50( \pm 0.86)$, representing $89.9 \%$ of the expected amount $(9.46 \mathrm{mg})$.

\subsubsection{Magnesium exchange}

Table 3 lists the metal concentrations in PAA samples after incubation of KPAA with $\mathrm{MgCl}_{2}$.

\subsubsection{Discussion}

Sodium, which is associated with the buffer PBS, was present in concentration comparable with that expected (in brackets when value can be calculated).

The sample containing aliquots 5-12 showed magnesium concentration lower than those of incubation solutions, due partially to the dilution during the sizeexclusion chromatography (the dilution is close to 10x).

As in the case of calcium, the percentage of bound magnesium confirm a saturation of PAA negative charges during exchange. Specifically, KPAA showed the potential to bind an amount of magnesium corresponding to $4.89 \%$ of its weight

\subsection{Binding properties of KPAA vs. Iron}

\subsubsection{Incubation procedure}

A volume of $5 \mathrm{~mL}$ of KPAA stock solution $\mathrm{n}$. 2 was incubated with $\mathrm{FeSO}_{4} \times 7 \mathrm{H}_{2} \mathrm{O}(38.8 \mathrm{mg}$, containing $7.79 \mathrm{mg}$ of $\mathrm{Fe}^{++}$) for 4 hours under stirring and then loaded onto the column for Size-Exclusion Chromatography to separate PAA (with or without bound metals) from free minerals. Figure 4 shows the elution profile of sizeexclusion chromatography. In this assay PAA was eluted in aliquots 6-12 and the chromatographic profile was different due to the strong absorbance of the red colour of the complex iron-PAA. The peak maximum is not visible since absorbance exceeded the limit of measure of the spectrophotometer. The fraction 6-12 was collected for further investigations.

\subsubsection{PAA concentration}

Using the linear regression described above (Fig. 1), the protein concentration in sample 6-12 was $1.285 \pm$ $0.027 \mathrm{mg} / \mathrm{mL}$; considering the total volume of this sample $(7 \mathrm{~mL})$, the $\mathrm{mg}$ of PAA recovered were $9.00( \pm 0.09)$, representing $95.1 \%$ of the expected amount $(9.46 \mathrm{mg})$. Thanks to the dilution of the sample, microbiuret method was applied without interference due to the red colour.

\subsubsection{Metal concentration}

Table 4 lists the results of metal quantification, after the incubation step between KPAA and $\mathrm{FeSO}_{4}$ and sizeexclusion chromatography.

\subsubsection{Discussion}

Sodium concentrations showed the same pattern observed in the previous incubations and were in agreement with the expected ones (in brackets when values can be calculated).

The sample containing aliquots 6-12 showed iron concentration lower than those of the incubation solution, due partially to the dilution during the size-exclusion chromatography.

The sample containing the aliquots 6-12 showed a percentage of iron lower than that of incubation solution (7.94 vs. $10.3 \%$ ). Also in this case saturation of negative charges was observed and KPAA showed an exchange potential for iron corresponding to $7.94 \%$ of its weight.

\section{Conclusions}

In conclusion, the assays performed in this study confirm the capability of polyaspartate to bind other cations. Table 5 summarized the binding observed and lists: a) the binding properties of KPAA for each mineral tested considering the highest dose of potassium polyaspartate 
Table 3. $\mathrm{Mg}$ and $\mathrm{Na}$ quantification in the sample $5-12^{\wedge}$.

\begin{tabular}{cccccc}
\hline Samples & PAA $(\mathbf{m g} / \mathbf{L})$ & $\mathbf{N a}(\mathbf{m g} / \mathbf{L})$ & $\mathbf{C a}(\mathbf{m g} / \mathbf{L})$ & $\mathbf{N a} / \mathbf{P A A} \%$ & $\mathbf{C a} / \mathbf{P A A} \%$ \\
\hline KPAA stock solution 1 & 9960 & 665 & 0.5 & 6.68 & 0.005 \\
\hline KPAA stock solution 2 & 9460 & 3911 & 0.7 & $41.3(39.2)$ & 0.007 \\
\hline KPAA stock solution 2 with added $\mathrm{MgCl}_{2}$ & 9460 & 4004 & 584 & $42.3(39.2)$ & $6.17(7.17)$ \\
\hline Aliquots 5-12 & 1063 & 3815 & 52 & $358.9(349)$ & 4.89 \\
\hline
\end{tabular}

${ }^{\wedge}$ In brackets the expected values

Table 4. Fe and Na quantification in the sample 6-12^.

\begin{tabular}{cccccc}
\hline Samples & PAA $(\mathbf{m g} / \mathbf{L})$ & $\mathbf{N a}(\mathbf{m g} / \mathbf{L})$ & $\mathbf{C a}(\mathbf{m g} / \mathbf{L})$ & Na/PAA \% & Ca/PAA \% \\
\hline KPAA stock solution 1 & 9960 & 665 & ND & 6.68 & - \\
\hline KPAA stock solution 2 & 9460 & 3911 & ND & $41.3(39.2)$ & - \\
\hline KPAA stock solution 2 with added $\mathrm{MgCl}_{2}$ & 9460 & 3732 & 976 & $39.45(39.2)$ & $10.32(16.47)$ \\
\hline Aliquots 6-12 & 1285 & 3022 & 102.1 & $235.2(290)$ & 7.94 \\
\hline
\end{tabular}

${ }^{\wedge}$ In brackets the expected values

$-\mathrm{ND}=$ Not Determined

Table 5. Interaction between KPAA and minerals and relative range of concentration in wine.

\begin{tabular}{|c|c|c|}
\hline Minerals & $\begin{array}{l}\text { Binding } \\
\text { exchange } \\
(\mathrm{mg} / \mathrm{L})^{*}\end{array}$ & $\begin{array}{c}\text { Natural minerals } \\
\text { concentration } \\
(\mathrm{mg} / \mathrm{L})[7]\end{array}$ \\
\hline $\mathrm{Ca}$ & 8.2 & $50-150$ \\
\hline$M g$ & 4.9 & $50-150$ \\
\hline $\mathrm{Fe}$ & 7.9 & $1-5$ \\
\hline$K$ & ND & $300-1500$ \\
\hline $\mathrm{Na}$ & ND & $5-60$ \\
\hline \multicolumn{2}{|c|}{ Total } & $406-1865$ \\
\hline
\end{tabular}

allowed in wines $(10 \mathrm{~g} / \mathrm{hL}$, corresponding to $100 \mathrm{mg} / \mathrm{L})$, and $b$ ) the range of natural content of the same minerals in wine $(\mathrm{mg} / \mathrm{L})$. The highest binding was observed with $\mathrm{Ca}$, where it reached $8.2 \%$ of PAA weight.

Wine in naturally rich in several minerals; their concentration depends mainly from soil where vines are grown, but other factors can influence it: the ripeness of the grapes, their variety, and the climate conditions [6].

According to the scientific literature, the amount of calcium, magnesium and iron naturally present in wine ranges between $50-150,50-150$ and $1-5 \mathrm{mg} / \mathrm{L}$, respectively (Table 5) [7]. In addition to these three minerals, wine contains also significative amounts of potassium (300-1500 mg/L) and sodium $(5-60 \mathrm{mg} / \mathrm{L})$.

It can be reasonably expected that when potassium polyaspartate is added into wine its binding capacity is saturated by the minerals naturally present in wine, so that the effect of the additive on the bioavailability of metals must be considered negligible.
Therefore, no nutrient depletion effects for consumers drinking wine treated with potassium polyaspartate at recommended dose of $100 \mathrm{mg} / \mathrm{L}$ wine is expected. This is a further demonstration of the safety of KPAA, when used as an additive for the tartaric stabilization of wine.

This research has been partially supported by the European Community's Seventh Framework Programme under Grant Agreement n. 314903 (STABIWINE Project). This paper does not reflect the Commission views or its future policy on this area.

\section{References}

[1] A. Bosso, L. Panero, M. Petrozziello, M. Sollazzo, A. Asproudi, S. Motta, M.M. Guaita, Food Chem. 185, 1 (2015)

[2] P. Ribéreau-Gayon, Y. Glories, A. Maujean, D. Dubourdieu, Handbook of Enology. Chichester, UK: John Wiley \& Sons Ltd. (2006)

[3] C. Galbusera, C. Casalegno, S. Marroncelli, G. Triulzi, J. Santos, E. Corsini, P. Restani, Food Chem. Toxicol. 109, 452 (2017)

[4] EFSA Opinion on KPAA, 2016. Safety of potassium polyaspartate (A-5D K/SD) for use as a stabiliser in wine. EFSA Panel on Food Additives and Nutrient Sources added to Food (ANS). EFSA J. 14 (3), 4435. https://doi.org/10.2903/j.efsa.2016.4435 adopted 9 March 2016, published 17 March 2016. www. efsa. europa.eu/ef sajournal

[5] R. F. Itzhaki, D. M. Gill, Anal. Biochem. 9, 401 (1964)

[6] P. Pohl, Analytical Chemistry 26, 9 (2007)

[7] M. Aceto, O. Abollino, M.C. Bruzzoniti, E. Entasti, C. Sarzanini, M. Malandrino, Food Addit. Contam. 19, $126(2002)$ 